\title{
The effects of hyperoxia liquid regulate cardiopulmonary bypass-induced myocardial damage through the Nrf2-ARE signaling pathway
}

\author{
XIAOCHUN MA ${ }^{1}$, BIN HU ${ }^{1}$, CHENGWEI ZOU ${ }^{1}$, AIGUO HAN ${ }^{2}$, \\ ZHENQIANG XU ${ }^{1}$, TAO ZHANG ${ }^{1}$ and WANCHENG YU ${ }^{1}$ \\ ${ }^{1}$ Department of Cardiovascular Surgery, Shandong Provincial Hospital Affiliated to Shandong University; \\ ${ }^{2}$ Department of Clinical Laboratory, The Fifth People's Hospital of Jinan, Jinan, Shandong 250021, P.R. China
}

Received December 27, 2017; Accepted June 6, 2018

DOI: $10.3892 / \mathrm{mmr} .2018 .9162$

\begin{abstract}
The aim of the present study was to investigate the protective role of hyperoxia liquid in regulating cardiopulmonary bypass (CPB)-induced myocardial damage and its possible underlying mechanism. In the CPB-induced rat model, hyperoxia liquid enhanced left ventricular ejection fraction (LVEF), reduced the left ventricular internal dimension systole (LVIDs) level, inhibited malondialdehyde levels, increased superoxide dismutase, glutathione (GSH) and GSH peroxidase levels, suppressed heart cell apoptosis, and induced the nuclear factor erythroid 2-related factor 2 (Nrf2) and heme oxygenase-1 (HO-1) signaling pathway. Then, ML385, a Nrf2 inhibitor, was used to attenuate the effect of hyperoxia liquid on LVEF and LVIDs levels, oxidative stress and heart cell apoptosis in the CPB-induced rat model. Collectively, the results of the present study demonstrated that the protective role of hyperoxia liquid may regulate oxidative stress in a CPB-induced rat model through the Nrf2-antioxidant response element signaling pathway.
\end{abstract}

\section{Introduction}

Hyperoxia liquids (HOS) is medical liquid containing high concentration of dissolved oxygen (DO). HOS is mainly prepared using the electrochemistry and rapid physical replacement of DO mechanisms. A large amount of $\mathrm{O}_{2}$ and little $\mathrm{O}_{3}$ are dissolved into the commonly used medical liquid in clinic. The oxygen partial pressure in the liquid is

Correspondence to: Mr. Bin Hu, Department of Cardiovascular Surgery, Shandong Provincial Hospital Affiliated to Shandong University, 324 Jingwuweiqi Road, Jinan, Shandong 250021, P.R. China

E-mail: hihtwmf105413@126.com

Key words: cardiopulmonary bypass, myocardial damage, hyperoxia liquid, nuclear factor erythroid 2-related factor 2, antioxidant response element
$153.7 \mathrm{mmHg}$ and the DO content is $3.8 \mathrm{mmg}$ before oxygen dissolving. However, the oxygen partial pressure has reached $795 \mathrm{mmHg}$ after oxygen dissolving. Meanwhile, its $\mathrm{O}_{2}$ content is 5.3-fold of that of conventional liquid, as well as 8.3 -fold and 21.3-fold of that in arterial and venous blood, respectively. The $\mathrm{O}_{3}$ concentration in the hyperoxic fluid measured immediately after oxygen dissolving using iodimetry is $26.5 \mu \mathrm{g} / \mathrm{ml}$. 20 min later, $\mathrm{O}_{3}$ is completely reduced to the dissolved $\mathrm{O}_{2}$. HOS can directly improve the blood oxygen partial pressure and blood DO content after it enters the blood. Meanwhile, it can indirectly enhance the chemically bonded oxygen (oxygen saturation). Currently, HOS is used in auxiliary oxygen supply treatment for multiple diseases with hypoxic symptoms. They include the first-aid in critical patients, cardiopulmonarycerebral resuscitation, central respiration repression, as well as severe head and face combined injury. In addition, it is also used in respiratory tract burn, pulmonary interstitial fibrosis, coal pneumoconiosis, pneumoconiosis, respiratory failure, one-lung ventilation during thoracic surgery, cerebral ischemia, myocardial ischemia, severe acute respiratory syndrome and high altitude hypoxia. Notably, favorable auxiliary effects have been attained.

Cardiopulmonary bypass (CPB) has been applied in cardiac and major vascular surgery for over half a century. Understanding towards CPB professional theoretical knowledge has been enhanced in recent years (1). In addition, medical materials, management level and surgical techniques have been improved (1). Therefore, the postoperative mortality and incidence of complications have been greatly reduced (2). This contributes to saving the lives of millions of patients. However, the CPB injury to the heart remains a research hotspot (2). According to literature report, the incidence of arrhythmia after CPB is related to underlying disease, surgical type and intraoperative myocardial protection (3). The incidence after surgery for congenital heart disease is $40-60 \%$, while that after valve replacement for valvular heart disease is $70-84 \%$. It has extended the length of stay in patients (3). Moreover, it will add to the incidence of complications and risk of death (4). Thus, it will cause tremendous economic burdens on both the family and the society. The mechanism of CPB in cardiac injury is quite complicated. It is mainly induced by cardiac ischemic 
damage, ventricular hyperinflation or emptiness, ascending aorta blocking and ischemia/reperfusion (I/R) injury (4).

Myocardial ischemia injury refers to abnormal myocardial energy metabolism, myocardial structural and functional changes derived (5) from reduced cardiac blood supply and hypoxidosis. Its pathogenesis is related to multi-layer and multi-target factors. Clinical and experimental studies suggest that oxidative stress plays a vital role in the genesis and development of myocardial ischemic injury diseases (6). Myocardial ischemia is accompanying with excessive production and accumulation of reactive oxygen species (ROS). It will induce endogenous oxidative stress and lipid peroxidation of unsaturated fatty acid on myocardial cell membrane (6). On the one hand, it will promote myocardial cell apoptosis and myocardial tissue necrosis. On the other hand, it will lead to changed cell membrane permeability and fluidity. Therefore, it will induce leakage of excessive intracellular myocardial enzymes and aggravate myocardial ischemia injury (7). Thus, effective intervention of the endogenous oxidative stress is an important pathway for improving myocardial ischemia injury and protecting myocardial tissue (7).

The nuclear factor erythroid 2-related factor 2 (Nrf2) pathway is the most important endogenous anti-oxidative stress pathway discovered recently (8). It plays a pivotal role in response to oxidative stress. Moreover, it is extensively distributed in the cardiovascular system (8). It is also closely related to improving myocardial ischemia injury. Generally, it exerts its anti-oxidative effect through two aspects (9). On the one hand, oxidative stress can promote Nrf 2 mRNA transcription and increase Nrf2 protein synthesis (8). On the other hand, Nrf2 dissociates with its chaperonin Keap1 in the case of electrophilic substance, ROS or upstream signaling pathway stimulation (8). As a result, increased amount of Nrf2 transfers from cytoplasm to cell nucleus. Subsequently, it binds with antioxidant response element (ARE) in the form of heterodimer with tendon fibrosarcoma protein $(8,10)$. Later, it up-regulates the expression of the downstream phase II detoxifying enzyme and antioxidase genes NQO1 and heme oxygenase-1 (HO-1). Thus, it can alleviate oxidative stress injury. HO-1 reduced prooxidant and attenuate cardiac hypertrophy through ROS production. Nrf2 upregulates HO-1 in different cell models to suppress oxidative stress in different cell. The protective role of hyperoxia liquid regulates $\mathrm{CPB}$-induced myocardial damage and its possible mechanism.

\section{Materials and methods}

Animals and treatment. Thirty adult male Sprague-Dawley rats (200-230 g) were purchased from the Center of Experimental Animal in Shandong Provincial Hospital Affiliated to Shandong University and were housed in individual cages with free access to food and water and maintained at an ambient temperature of $22 \pm 2^{\circ} \mathrm{C}$, on a $12-\mathrm{h}$ light/dark cycle (8:00 a.m.-8:00 p.m.). All rats $(n=18)$ were randomly allocated into three groups: control $(n=6)$, model $(n=6)$ and Hyperoxia liquids group $(n=6)$. Pre-treatment with $15 \mathrm{ml} / \mathrm{kg}$ hyperoxia liquids for 7 day was intravenous injected into rat of hyperoxia liquids group. After 7 day, rat of model or hyperoxia liquids were anesthetized i.p. with $30 \mathrm{mg} / \mathrm{kg}$ sodium pentobarbital (Sigma-Aldrich; Merck KGaA, Darmstadt, Germany). Heparin (150 U) and vecuronium bromide $(2 \mathrm{mg} / \mathrm{kg}$ IV) was induced by switching off the ventilator for $30 \mathrm{~min}$. Resuscitation was started with the initiation of CPB flow and resumption of ventilation. The slipknot was released and rat was received $120 \mathrm{~min}$ of reperfusion.

Next, all rats were randomly allocated into four groups $(n=24)$ : Control $(n=6)$, model $(n=6)$, Hyperoxia liquids group $(n=6)$ and ML385 $(n=6)$. In ML385 group, pre-treatment with $15 \mathrm{ml} / \mathrm{kg}$ Hyperoxia liquids and $5 \mathrm{mg} / \mathrm{kg}$ of ML385 for 7 day was intravenous injected into rat of hyperoxia liquids group. Other experimental procedure was invariability. The present study was approved by the Ethical Approval Committee of Shandong Provincial Hospital Affiliated to Shandong University (Shandong, China).

Detection of cardiac function. Rats were anesthetized with $30 \mathrm{mg} / \mathrm{kg}$ sodium pentobarbital and a catheter was inserted into the left ventricle, and which was used to measure left ventricular ejection fraction (LVEF) and left ventricular internal dimension systole (LVIDs).

Measurement of serum oxidative stress and tissue caspase-3/8/9 activity. Serum was collected by centrifugation at $1,000 \mathrm{x} \mathrm{g}$ for $10 \mathrm{~min}$ at $4^{\circ} \mathrm{C}$ and used to analyze the levels of malondialdehyde (MDA; S0131), superoxide dismutase (SOD; S0101), glutathione (GSH; S0053), glutathione-peroxidase (GSH-PX; S0056) using ELISK kits (Beyotime Institute of Biotechnology, Nanjing, China). Total proteins of the cell were extracted by using RIPA assay and Protein concentration was estimated by BCA assay. Protein $(10 \mu \mathrm{g})$ was used to analyze caspase-3/8/9 activity using caspase-3/8/9 activity kits (C1116, C1152, C1158; Beyotime Institute of Biotechnology).

Western blot analysis. Total proteins of the cell were extracted by using RIPA assay and Protein concentration was estimated by BCA assay. Protein $(30 \mu \mathrm{g})$ was loaded for $10 \%$ SDS-polyacrylamide gel electrophoresis and transferred onto polyvinylidene difluoride membranes. The membranes were blocked with 5\% non-fat milk in TBS and incubated with Nrf2 (sc-722, 1:1,000; Santa Cruz Biotechnology, Inc., Dallas, TX, USA), HO-1 (sc-10789, 1:1,000; Santa Cruz Biotechnology, Inc.) and GAPDH (sc-25778, 1:1,000; Santa Cruz Biotechnology, Inc.) at $4^{\circ} \mathrm{C}$ overnight. The membranes were washed with TBS containing $0.1 \%$ Tween-20 and incubated with horseradish peroxidase-conjugated secondary antibodies (sc-2004, 1:5,000; Santa Cruz Biotechnology, Inc.) for $1 \mathrm{~h}$. Protein expression were detected by Amersham ECL Plus Western Blotting Detection kit (GE Healthcare Life Sciences, Piscataway, NJ, USA) and analyzed using Image-ProPlus 6.0 software (Media Cybernetics, Inc., Rockville, MD, USA).

Statistical analysis. Results are expressed as the mean \pm standard error of the mean. Statistical analysis was performed using SPSS 17.0 software (SPSS, Inc., Chicago, IL, USA). Differences between groups were calculated using one-way analysis of variance with Tukey's post-hoc test. $\mathrm{P}<0.05$ was considered to indicate a statistically significant difference. 
A

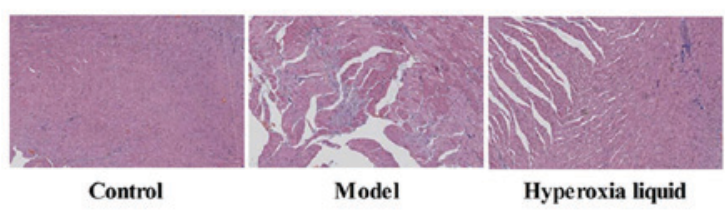

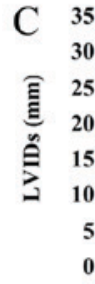

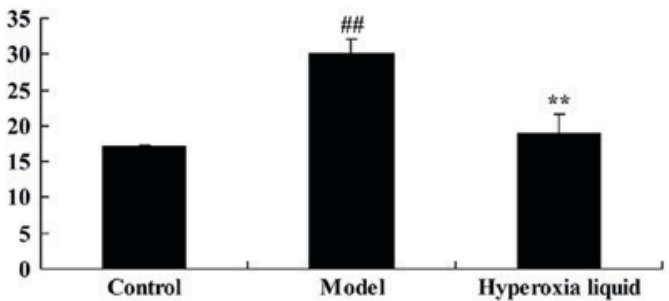

E
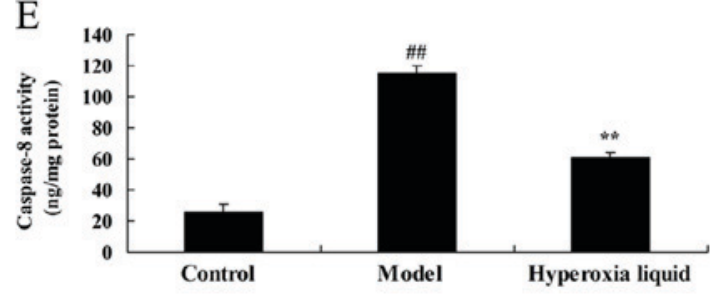
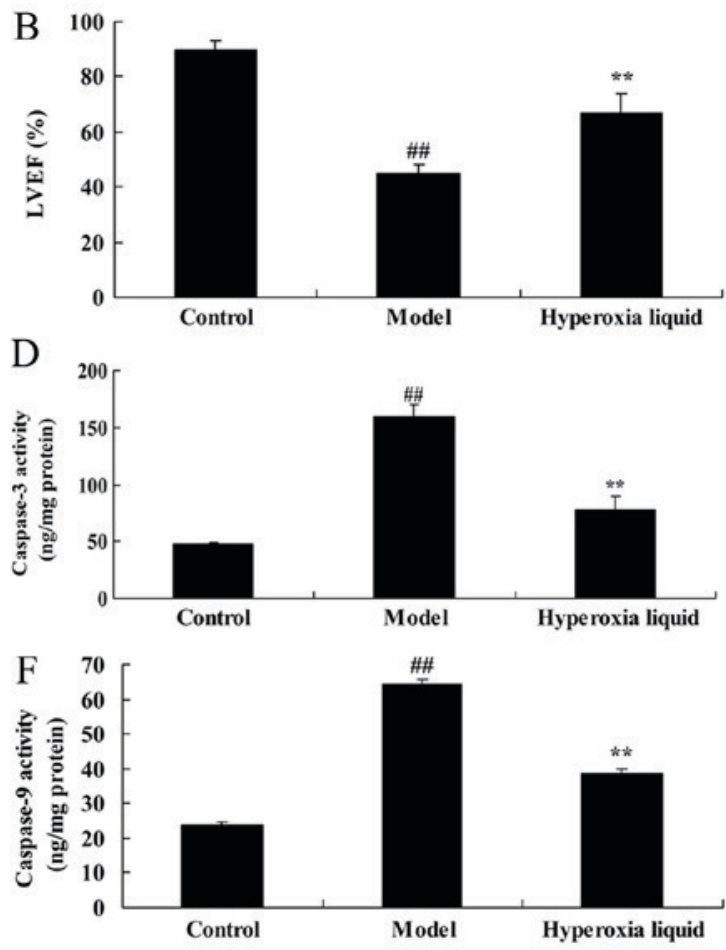

Figure 1. Effects of hyperoxia liquid regulation on heart cell apoptosis in a CPB-induced rat model. (A) Hematoxylin and eosin staining of heart tissues (magnification, x5). Levels of (B) LVEF, (C) LVIDs, and (D) caspase-3, (E) caspase-8 and (F) caspase-9 activity. ${ }^{\# \#} \mathrm{P}<0.01$ vs. control group; ${ }^{* *} \mathrm{P}<0.01$ vs. CPB induced rat model group. CPB, cardiopulmonary bypass; Control, control normal rat group; Model, CPB induced rat model group; Hyperoxia liquid, treatment with hyperoxia liquid group; LVEF, left ventricular ejection fraction; LVIDs, left ventricular internal dimension systole.

\section{Results}

The effects of hyperoxia liquid regulated heart cell apoptosis in $C P B$ induced rat model. Our study investigated the effects of hyperoxia liquid in regulating heart cell apoptosis in $\mathrm{CPB}$ induced rat model. H\&E staining showed that heart cell apoptosis in CPB induced rat model was higher than that of control group (Fig. 1A). The effects of hyperoxia liquid inhibited heart cell apoptosis in CPB rat, compared with CPB induced rat model group (Fig. 1A). The LVEF was inhibited, and LVIDs level was increased in CPB induced rat model, compared with CPB induced rat model group (Fig. 1B and C). Hyperoxia liquid enhanced LVEF and reduced LVIDs level in CPB induced rat model, compared with CPB rat (Fig. 1B and C). Caspase-3/8/9 activity levels in CPB were elevated, compared with control group (Fig. 1D-F). In addition, hyperoxia liquid reduced the CPB-induced Caspase-3/8/9 activity levels in rat, compared with CPB induced rat model (Fig. 1D-F).

The effects of hyperoxia liquid regulated oxidative stress in $C P B$ induced rat model. We analyzed the effects of hyperoxia liquid on the changes of oxidative stress in $\mathrm{CPB}$ induced rat model. As shown in Fig. 2, MDA level was increased, and SOD, GSH, GAH-PX levels were decreased in CPB, compared with control group. Moreover, the promotion of MDA level, and the inhibition of SOD, GSH, GAH-PX levels were reversed after administration with hyperoxia liquid, compared with CPB induced rat model (Fig. 2).

The effects of hyperoxia liquid induced Nrf2-ARE signaling pathway in CPB induced rat model. To confirm the mechanism of hyperoxia liquid on oxidative stress in $\mathrm{CPB}$ induced rat model, Nrf2-ARE signaling pathway was analyzed using Western blot. As was shown in Fig. 3, Nrf2 and HO-1 protein expressions were suppressed, while Bax protein expression was induced by hyperoxia liquid in CPB induced rat model, compared with control group. Administration with hyperoxia liquid induced Nrf2 and HO-1 protein expressions, and suppressed Bax protein expression in CPB induced rat model, compared with CPB induced rat model (Fig. 3).

The inhibition of Nrf2 reduced the function of hyperoxia liquid on Nrf2-ARE signaling pathway in CPB induced rat model. Next, ML385, an Nrf2 inhibitor, was utilized to reduce the function of hyperoxia liquid on Nrf2-ARE signaling pathway in CPB induced rat model. As shown in Fig. 4, Nrf2 inhibitor suppressed $\mathrm{Nrf} 2$ and $\mathrm{HO}-1$ protein expressions, and induced Bax protein expression in CPB induced rat, compared with CPB induced rat model. Treatment with Nrf2 inhibitor suppressed the function of hyperoxia liquid on heart cell apoptosis, the inhibition of LVEF level, promotion of LVIDs level, and caspase-3/8/9 activity levels in CPB induced rat, compared CPB induced rat model (Fig. 5). Moreover, the inhibition of $\mathrm{Nrf} 2$ reduced the function of hyperoxia liquid on the promotion of MDA, and the inhibition of SOD, GSH, GAH-PX levels in CPB induced rat, compared with CPB induced rat model (Fig. 6).

\section{Discussion}

The myocardial protection technique in hypothermic CPB on-pump open-heart surgery has gradually emerged in recent 

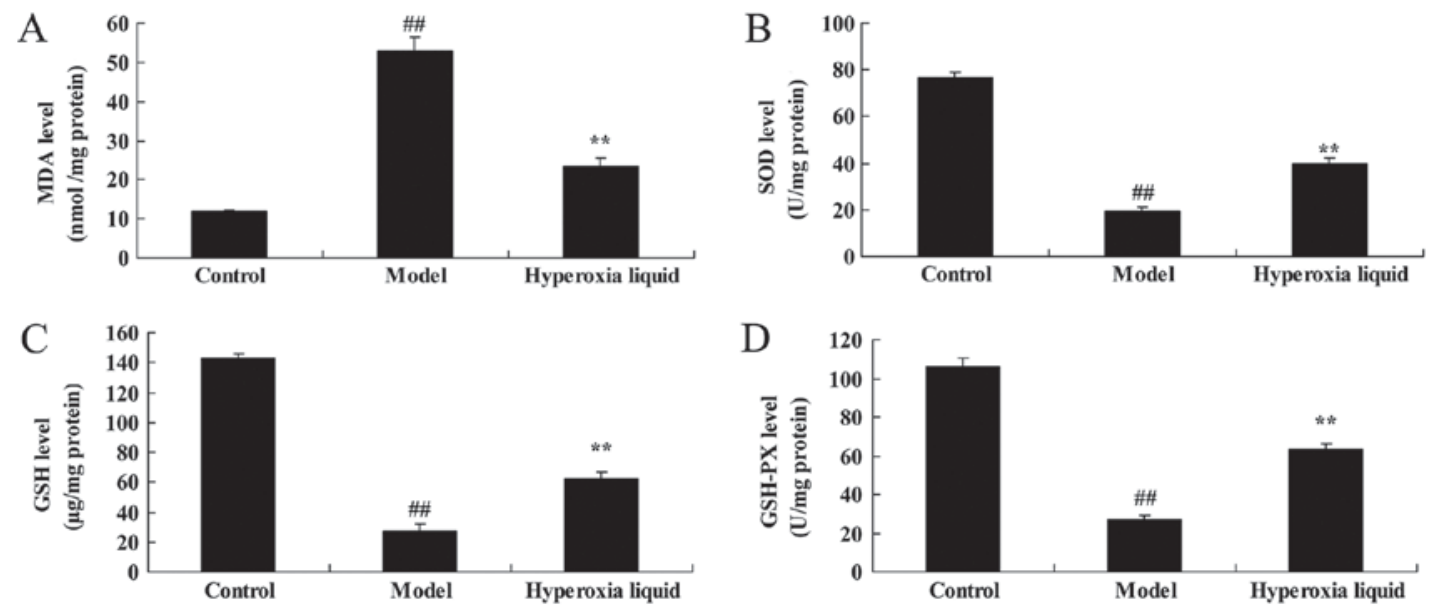

Figure 2. Effects of hyperoxia liquid on the regulation of oxidative stress in the CPB-induced rat model. Levels of (A) MDA, (B) SOD, (C) GSH and (D) GAH-PX. ${ }^{\# \#} \mathrm{P}<0.01$ vs. control group; ${ }^{* *} \mathrm{P}<0.01$ vs. CPB induced rat model group. CPB, cardiopulmonary bypass; Control, control normal rat group; Model, CPB induced rat model group; Hyperoxia liquid, treatment with hyperoxia liquid group; MDA, malondialdehyde; SOD, superoxide dismutase; GSH, glutathione; GSH-PX, glutathione-peroxidase.

A
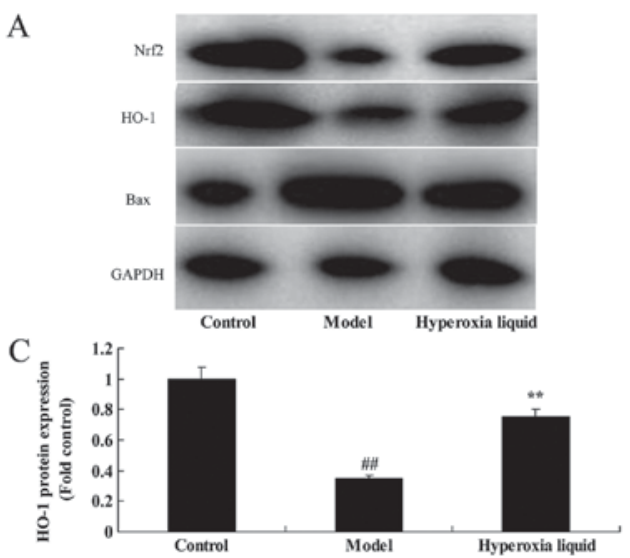

B
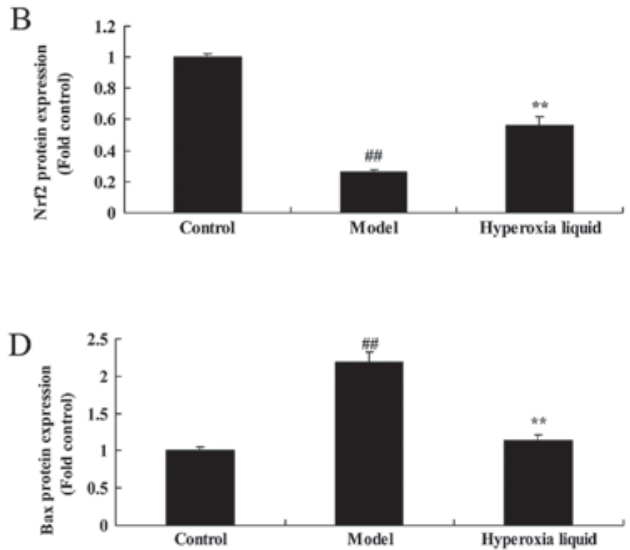

Figure 3. Effects of hyperoxia liquid on the Nrf2-ARE signaling pathway in the CPB-induced rat model. (A) Western blotting for Nrf2, HO-1, Bax and GAPDH protein expression, and the statistical analysis of (B) Nrf2, (C) HO-1 and (D) Bax protein expression. GAPDH was used as the internal control. ${ }^{\# \#} \mathrm{P}<0.01$ vs. control group; ${ }^{* *} \mathrm{P}<0.01$ vs. CPB induced rat model group. $\mathrm{CPB}$, cardiopulmonary bypass; Control, control normal rat group; Model, $\mathrm{CPB}$ induced rat model group; Hyperoxia liquid, treatment with hyperoxia liquid group; Nrf2, nuclear factor erythroid 2-related factor 2; ARE, antioxidant response element; HO-1, heme oxygenase 1; Bax, B-cell lymphoma-associated X protein.

A
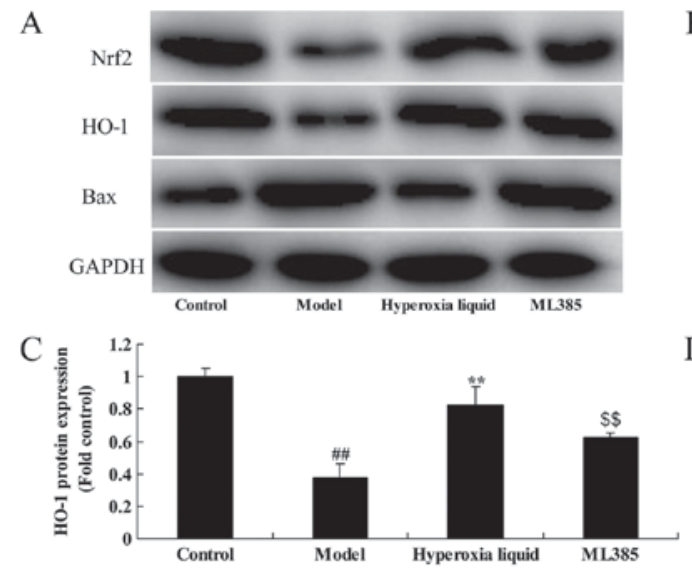
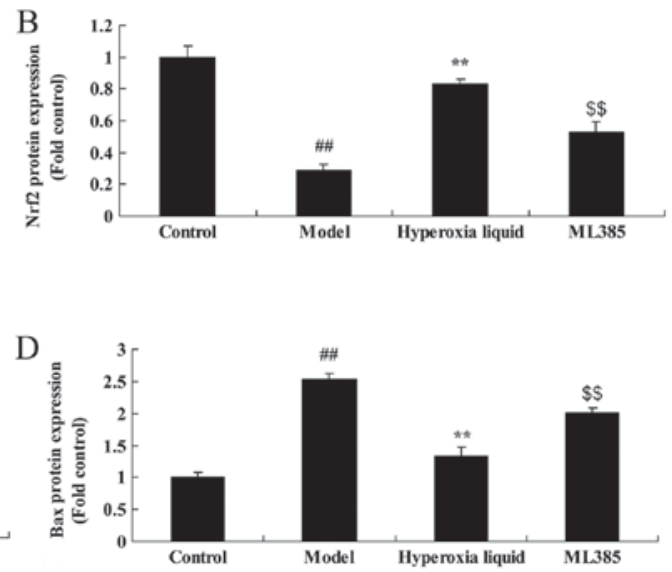

Figure 4. Inhibition of Nrf2 reduces the effect of hyperoxia liquid on the Nrf2-ARE signaling pathway in the CPB-induced rat model. (A) Western blotting for Nrf2, HO-1, Bax and GAPDH protein expression, and the statistical analysis of (B) Nrf2, (C) HO-1 and (D) Bax protein expression, following treatment with ML385. GAPDH was used as the internal control. ${ }^{\# \#} \mathrm{P}<0.01$ vs. control group; ${ }^{* *} \mathrm{P}<0.01$ vs. CPB induced rat model group; ${ }^{\$ \$} \mathrm{P}<0.01$ vs. Hyperoxia liquid group. $\mathrm{CPB}$, cardiopulmonary bypass; Control, control normal rat group; Model, CPB induced rat model group; Hyperoxia liquid, treatment with hyperoxia liquid group; ML385, treatment with hyperoxia liquid and ML385 group; Nrf2, nuclear factor erythroid 2-related factor 2; ARE, antioxidant response element; HO-1, heme oxygenase 1; Bax, B-cell lymphoma-associated X protein. 
A
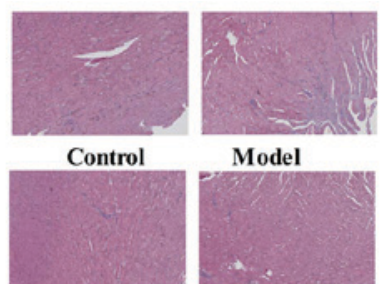

Hyperoxia

liquid

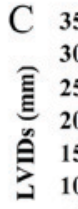

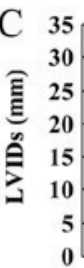
Model

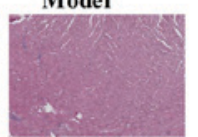

ML385
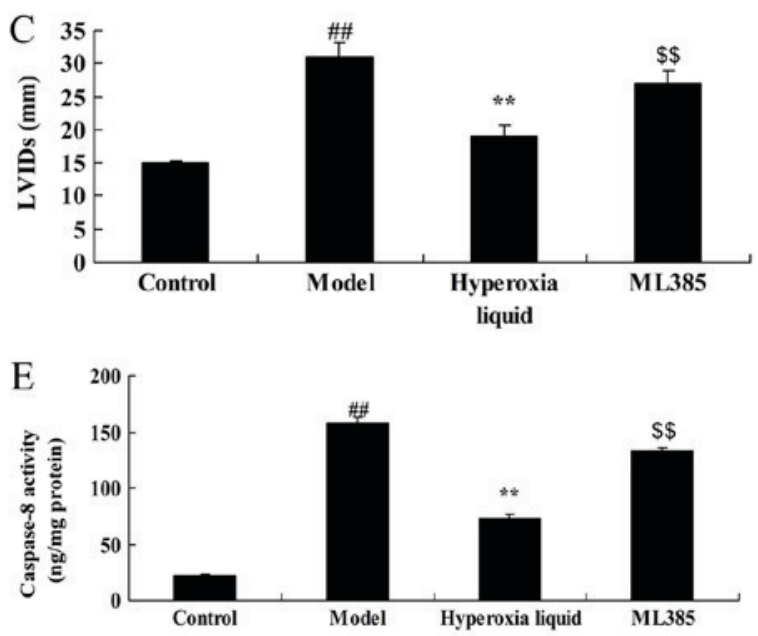
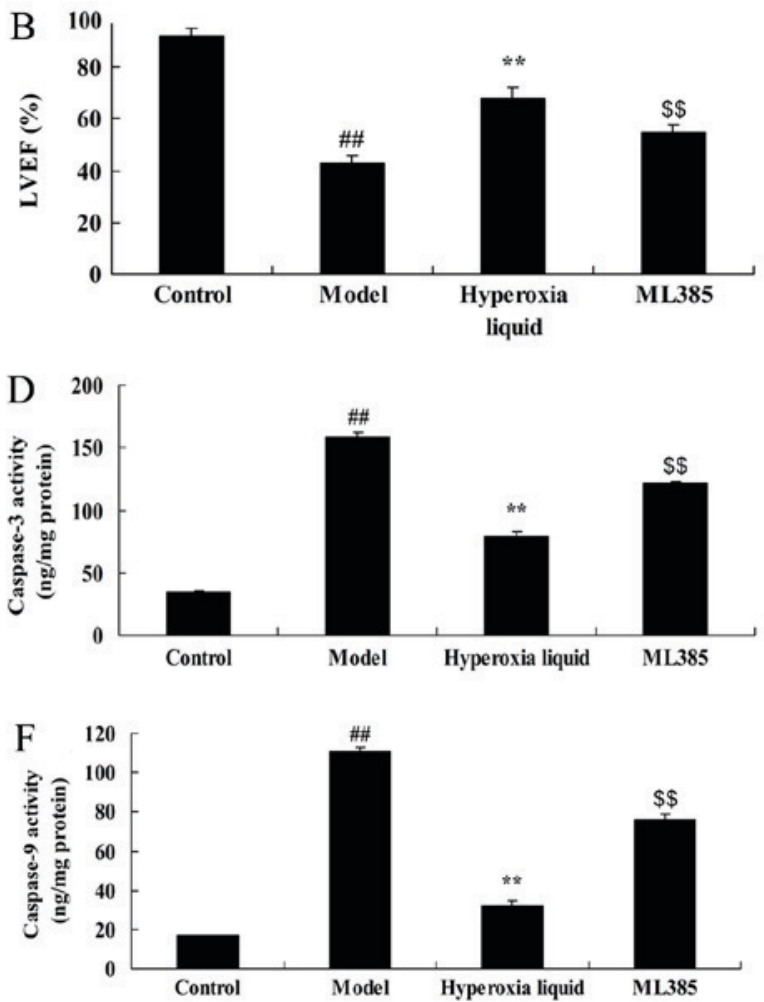

Figure 5. Inhibition of nuclear factor erythroid 2-related factor 2 reduced the effect of hyperoxia liquid on heart cell apoptosis in the CPB-induced rat model. (A) Hematoxylin and eosin staining for heart tissue (magnification, x5). Levels of (B) LVEF, (C) LVIDs, and (D) caspase-3, (E) -8 and (F) -9 activity. ${ }^{\# \#} \mathrm{P}<0.01$ vs. control group; ${ }^{* *} \mathrm{P}<0.01$ vs. $\mathrm{CPB}$ induced rat model group; ${ }^{\$ \$} \mathrm{P}<0.01$ vs. Hyperoxia liquid group. CPB, cardiopulmonary bypass; Control, control normal rat group; Model, $\mathrm{CPB}$ induced rat model group; Hyperoxia liquid, treatment with hyperoxia liquid group; ML385, treatment with hyperoxia liquid and ML385 group; LVEF, left ventricular ejection fraction; LVIDs, left ventricular internal dimension systole.
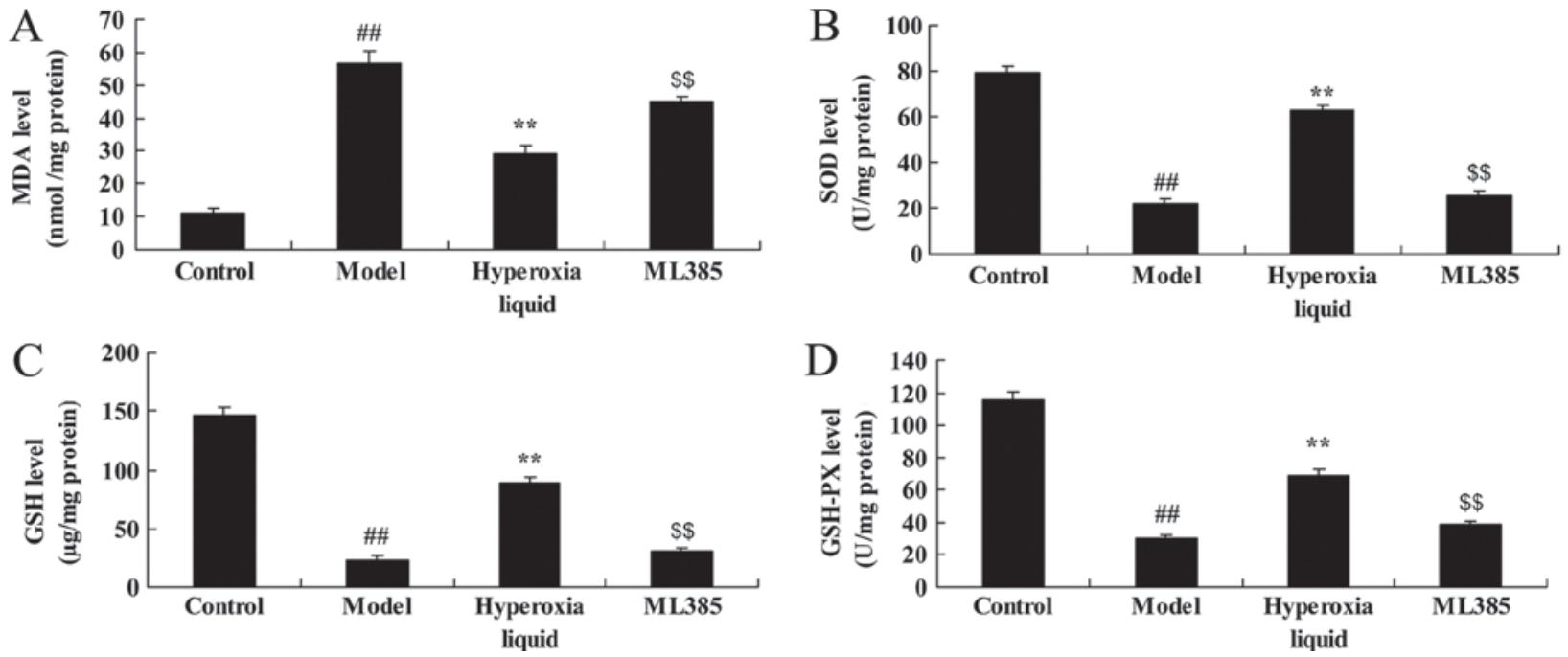

Figure 6. Inhibition of Nrf2 reduces the effect of hyperoxia liquid on oxidative stress in the CPB-induced rat model. Levels of (A) MDA, (B) SOD, (C) GSH and (D) GAH-PX, following ML385 treatment. ${ }^{\# \#} \mathrm{P}<0.01$ vs. control group; ${ }^{* *} \mathrm{P}<0.01$ vs. CPB induced rat model group; ${ }^{\$ \$} \mathrm{P}<0.01$ vs. Hyperoxia liquid group. $\mathrm{CPB}$, cardiopulmonary bypass; Control, control normal rat group; Model, CPB induced rat model group; Hyperoxia liquid, treatment with hyperoxia liquid group; ML385, treatment with hyperoxia liquid and ML385 group; MDA, malondialdehyde; SOD, superoxide dismutase; GSH, glutathione; GSH-PX, glutathione-peroxidase.

years (11). Favorable effects have been achieved in clinic (12). Meanwhile, it is associated with great drawbacks, such as poor exposure, increased blood return in surgical field and elevated difficulty in surgical operation (12). In addition, it will lead to increased intracardiac suction, further aggravating the destruction of visible blood components. It will also increase the risk of gas embolism (12). Meanwhile, ventricular fibrillation may be induced intraoperatively due to the low temperature. CPB myocardial injury is associated with high incidence of arrhythmia. Moreover, the damage mechanism is complicated, which has involved diverse influence factors. These have proposed higher challenges 


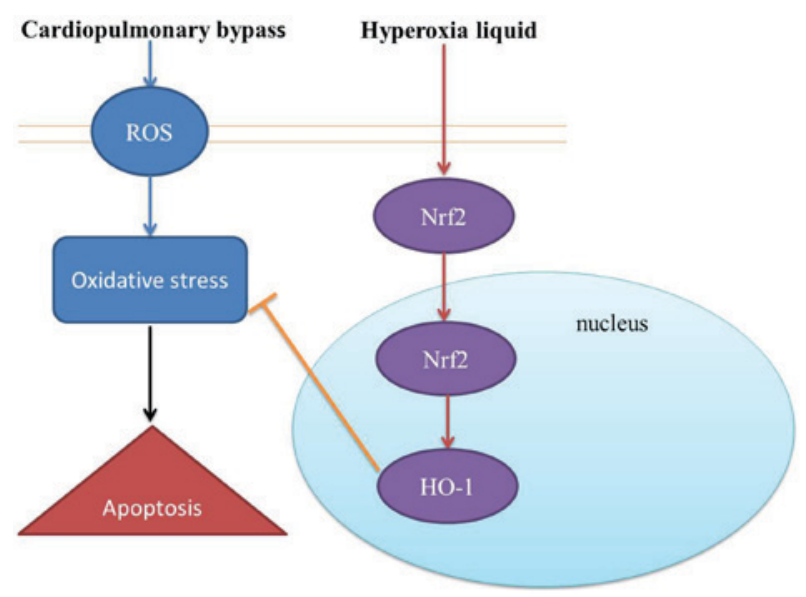

Figure 7. Effects of hyperoxia liquid regulation of cardiopulmonary bypass-induced myocardial damage through the Nrf2-ARE signaling pathway. ROS, reactive oxygen species; Nrf2, nuclear factor erythroid 2-related factor 2; HO-1, heme oxygenase 1.

and requirements for clinical prevention and treatment of cardiac surgery. The above measures have been comprehensively applied. However, no expected clinical effect can be attained (12). Therefore, CPB perioperative myocardial protection remains a research hotspot at present and in the future. In the present study, we demonstrated that the effects of hyperoxia liquid inhibited heart cell apoptosis in CPB induced rat model. Gao et al (13) showed that therapeutic effects of hyperoxia liquid improve systemic oxygenation during one-lung ventilation.

Free radical scavenger refers to substance that can delay, inhibit and block the active oxygen/oxygen radical oxidative injury (14). It can bind with and scavenge oxygen radicals (15). ATP reserve in myocardial tissue is reduced during the early myocardial ischemia. Subsequently, it will provide energy through anaerobic metabolism (14). In early reperfusion, blood flow oxygen cannot be sufficiently used due to the recovery of coronary blood flow (14). This results in the production of oxygen radicals with potential damage by various oxidase substrates. It leads to excessive accumulation of free radicals. This will then attach the unsaturated fatty acid in membrane phospholipid (16). Later, it will induce changes in fluidity and permeability of myocardial cell membrane and subcellular organelle membrane (15). Then, multiple lipid peroxides will be produced, affecting the myocardial cell integrity and function. In addition, it will attack the structural protein of myocardial cell, rendering cleavage of peptide-chain (17). Oxidative stress is particularly important in CPB and myocardial damage. In the present study, resveratrol was suggested the effects of hyperoxia liquid reduced oxidative stress in CPB induced rat model. Zhao et al (18) suggested that hyperoxia liquid protects against acute hypobaric hypoxia-induced oxidative damage. Karu et al (19) reported that the effects of $60 \mathrm{~min}$ of hyperoxia followed by normoxia before coronary artery reduced myocardial injury and inflammatory response profile.

The Nrf2-ARE signaling pathway is recognized to be the most important endogenous anti-oxidative stress pathway (20). Activation of this pathway can induce the production of antioxidant enzyme and phase II drug metabolic enzyme (21). It plays a vital role in maintaining intracellular redox state, reducing oxidative damage and protecting cell function. Research finds that the Nrf2-ARE signaling pathway is closely related to improving myocardial I/R injury. Meanwhile, Nrf2 is an important transcription factor in cell to reduce ROS (22). Our results suggest that the effects of hyperoxia liquid induced Nrf2-ARE signaling pathway in CPB induced rat model. Nrf2 inhibitor, reduced the function of hyperoxia liquid in CPB induced rat model. Karu et al (23) showed that pre-treatment with hyperoxia before coronary artery bypass grafting - effects on myocardial injury and inflammatory response. Nagato et al (24) showed that Hyperoxia promotes polarization of the immune response, and induced Nrf2 and iNOS protein expression. Šarić et al (25) showed that this model of hyperoxia as a useful tool to assess sex differences in adaptive response to acute stress conditions through activation of HO-1 and Nrf2 protein expression. These experiments are three independent experiments, tendency is correct.

Taken together, the results of this study suggest that the effects of hyperoxia liquid inhibited oxidative stress to open heart apoptosis in CPB-induced myocardial damage through Nrf2-ARE signaling pathway (Fig. 7). Our present results represent useful tools for the development of a new mode for treatment of CPB-induced myocardial damage.

\section{Acknowledgements}

Not applicable.

\section{Funding}

The present study was supported by grants from the Research Award Fund for Outstanding Young-Middle Aged Scientists of Shandong Province (grant no. BS2014YY004) and Natural Science Foundation of Shandong Province (grant no. ZR2017BH017).

\section{Availability of data and materials}

The analyzed datasets generated during the study are available from the corresponding author on reasonable request.

\section{Authors' contributions}

BH designed the experiments. XM, CZ, AH, ZX, TZ and WY performed the experiments. $\mathrm{BH}$ and $\mathrm{XM}$ analyzed the data, and $\mathrm{BH}$ wrote the manuscript.

\section{Ethics approval and consent to participate}

The present study was approved by the Ethical Approval Committee of Shandong Provincial Hospital Affiliated to Shandong University (Shandong, China).

\section{Patient consent for publication}

Not applicable.

\section{Competing interests}

The authors declare that they have no competing interests. 


\section{References}

1. Ching T, Song MA, Tiirikainen M, Molnar J, Berry M, Towner D and Garmire LX: Genome-wide hypermethylation coupled with promoter hypomethylation in the chorioamniotic membranes of early onset pre-eclampsia. Mol Hum Reprod 20: 885-904, 2014.

2. Wang G, Xiao S and Gao C: The effects of cardiopulmonary bypass on pulmonary function during robotic cardiac surgery. Perfusion 30: 213-218, 2015.

3. Wang X, Ji B, Zhang Y, Zhu X, Liu J, Long C and Zheng Z: Comparison of the effects of three cell saver devices on erythrocyte function during cardiopulmonary bypass procedure-a pilot study. Artif Organs 36: 931-935, 2012.

4. Arsenault KA, Paikin JS, Hirsh J, Dale B, Whitlock RP, Teoh K, Young E, Ginsberg JS, Weitz JI and Eikelboom JW: Subtle differences in commercial heparins can have serious consequences for cardiopulmonary bypass patients: A randomized controlled trial J Thorac Cardiovasc Surg 144: 944-950. e3, 2012.

5. Lee D, Park S, Bae S, Jeong D, Park M, Kang C, Yoo W, Samad MA, Ke Q, Khang G and Kang PM: Hydrogen peroxide-activatable antioxidant prodrug as a targeted therapeutic agent for ischemia-reperfusion injury. Sci Rep 5: 16592, 2015.

6. Liu H, Jing X, Dong A, Bai B and Wang H: Overexpression of TIMP3 protects against cardiac ischemia/reperfusion injury by inhibiting myocardial apoptosis through ROS/Mapks pathway. Cell Physiol Biochem 44: 1011-1023, 2017.

7. Hirji SA, Stevens SR, Shaw LK, Campbell EC, Granger CB, Patel MR, Sketch MH Jr, Wang TY, Ohman EM, Peterson ED and Brennan JM: Predicting risk of cardiac events among ST-segment elevation myocardial infarction patients with conservatively managed non-infarct-related artery coronary artery disease: An analysis of the Duke Databank for Cardiovascular disease. Am Heart J 194: 116-124, 2017.

8. Wang $\mathrm{J}, \mathrm{Hu} \mathrm{X}$ and Jiang $\mathrm{H}$ : ERS-PERK signaling pathway-mediated Nrf2/ARE-HO-1 axis: A novel therapeutic target for attenuating myocardial ischemia and reperfusion injury. Int J Cardiol 203: 779-780, 2016.

9. Lee TM, Lin SZ and Chang NC: Antiarrhythmic effect of lithium in rats after myocardial infarction by activation of $\mathrm{Nrf} 2 / \mathrm{HO}-1$ signaling. Free Radic Biol Med 77: 71-81, 2014.

10. Li W, Wu M, Tang L, Pan Y, Liu Z, Zeng C, Wang J, Wei T and Liang G: Novel curcumin analogue $14 \mathrm{p}$ protects against myocardial ischemia reperfusion injury through Nrf2-activating anti-oxidative activity. Toxicol Appl Pharmacol 282: 175-183, 2015.

11. Tashiro N, Takahashi S, Takasaki T, Katayama K, Taguchi T, Watanabe M, Kurosaki T, Imai K, Kimura $H$ and Sueda T: Efficacy of cardiopulmonary rehabilitation with adaptive servo-ventilation in patients undergoing off-pump coronary artery bypass grafting. Circ J 79: 1290-1298, 2015.

12. Elgebaly AS and Sabry M: Infusion of low-dose vasopressin improves left ventricular function during separation from cardiopulmonary bypass: A double-blind randomized study. Ann Card Anaesth 15: 128-133, 2012.
13. Gao C, Zhang G, Sun X, Zhang H, Kuai J, Zhao H, Yao L, Yu D, Yang Y, Xu L and Chai W: The effects of intravenous hyperoxygenated solution infusion on systemic oxygenation and intrapulmonary shunt during one-lung ventilation in pigs. J Surg Res 159: 653-659, 2010

14. Eigel BN, Gursahani H and Hadley RW: ROS are required for rapid reactivation of $\mathrm{Na}+\mathrm{Ca} 2+$ exchanger in hypoxic reoxygenated guinea pig ventricular myocytes. Am J Physiol Heart Circ Physiol 286: H955-H963, 2004.

15. Kayama Y, Raaz U, Jagger A, Adam M, Schellinger IN, Sakamoto M, Suzuki H, Toyama K, Spin JM and Tsao PS: Diabetic cardiovascular disease induced by oxidative stress. Int J Mol Sci 16: 25234-25263, 2015.

16. Yuan Y, Pan S, Yang SL, Liu YL and Xu QM: Antioxidant and cardioprotective effects of Ilex cornuta on myocardial ischemia injury. Chin J Nat Med 15: 94-104, 2017.

17. Bashar T and Akhter N: Study on oxidative stress and antioxidant level in patients of acute myocardial infarction before and after regular treatment. Bangladesh Med Res Counc Bull 40: 79-84, 2014.

18. Zhao H, Chai W, Gao W, Xu L, Zhang H and Yang Y: Hyperoxygenated solution: Effects on acute hypobaric hypoxia-induced oxidative damage in rabbits. High Alt Med Biol 10: 283-291, 2009.

19. Karu I, Tähepõld P, Ruusalepp A, Zilmer K, Zilmer M and Starkopf J: Effects of 60 min of hyperoxia followed by normoxia before coronary artery bypass grafting on the inflammatory response profile and myocardial injury. J Negat Results Biomed 11: 14, 2012

20. Gao S, Zhan L, Yang Z, Shi R, Li H, Xia Z, Yuan S, Wu QP, Wang $\mathrm{T}$ and Yao S: Remote limb ischaemic postconditioning protects against myocardial ischaemia/reperfusion injury in mice: Activation of JAK/STAT3-mediated Nrf2-antioxidant signalling. Cell Physiol Biochem 43: 1140-1151, 2017.

21. Donnarumma E, Bhushan S, Bradley JM, Otsuka H, Donnelly EL, Lefer DJ and Islam KN: Nitrite therapy ameliorates myocardial dysfunction via $\mathrm{H} 2 \mathrm{~S}$ and nuclear factor-erythroid 2-related factor 2 (Nrf2)-dependent signaling in chronic heart failure. J Am Heart Assoc 5: pii: e003551, 2016.

22. Zhang X, Hu H, Luo J, Deng H, Yu P, Zhang Z, Zhang G, Shan L and Wang Y: A novel danshensu-tetramethylpyrazine conjugate DT-010 provides cardioprotection through the PGC-1 $\alpha /$ Nrf2/HO-1 pathway. Biol Pharm Bull 40: 1490-1498, 2017.

23. Karu I, Loit R, Zilmer K, Kairane C, Paapstel A, Zilmer M and Starkopf J: Pre-treatment with hyperoxia before coronary artery bypass grafting-effects on myocardial injury and inflammatory response. Acta Anaesthesiol Scand 51: 1305-1313, 2007.

24. Nagato AC, Bezerra FS, Talvani A, Aarestrup BJ and Aarestrup FM: Hyperoxia promotes polarization of the immune response in ovalbumin-induced airway inflammation, leading to a TH17 cell phenotype. Immun Inflamm Dis 3: 321-337, 2015.

25. Šarić A, Sobočanec S, Mačak Šafranko Ž, Popović Hadžija M, Bagarić R, Farkaš V, Svarc A, Marotti T and Balog T: Diminished resistance to hyperoxia in brains of reproductively senescent female CBA/H mice. Med Sci Monit Basic Res 21: 191-199, 2015. 